\title{
Editorial
}

\section{Bibliometría e indicadores no convencionales}

Las editoriales presentadas en el volumen 44 (2021) de la Revista Técnica de la Facultad de Ingeniería de LUZ, versaron sobre distintas aristas que componen la evaluación del desarrollo científico-técnico en Latinoamérica. En el nro. 1 la Dra. Valentina Millano (Universidad del Zulia, Venezuela), escribió sobre el acceso abierto y los sistemas de evaluación de la ciencia, ¿calidad invisibilizada vs. cantidad comercializada?; mientras que en el nro. 2 la Dra. Nilda Rosales (Universidad de Los Andes, Venezuela), con una mirada a las universidades venezolanas a través de los rankings académicos, destaca que los datos son preocupantes, pues la caída en la publicación de artículos científicos es sostenida, así como la pérdida de posicionamiento internacional. Para este nro. 3 se presenta un esbozo acerca de la bibliometría y la necesidad de crear indicadores no convencionales para publicaciones científico-técnicas latinoamericanas.

Hoy en día la bibliometría se entiende como una herramienta o disciplina que funge como método de análisis y medición de los documentos científicos; tiene carácter interdisciplinar y su evolución ha sido signada por la producción científica especializada (Peralta et al., 2015). El crecimiento de este tipo de literatura en las últimas décadas y su recopilación en bases de datos bibliográficas automatizadas, han potenciado el uso de la bibliometría y la generación de indicadores para medir los resultados de la actividad científica y tecnológica. Los indicadores bibliométricos son datos estadísticos deducidos de las distintas características de las publicaciones científicas, con base en el papel preponderante que desempeñan estas en la difusión y transmisión del conocimiento generado en la investigación (De Filippo y Fernández, 2003). Los indicadores utilizados en los estudios bibliométricos dependen de múltiples factores, en respuesta a la gran variedad de propuestas existentes y su aplicación a diferentes casos de estudio. Entre los más usados por los distintos índices internacionales, se encuentran: productividad, colaboración, multiautoría, institucionalidad, transitoriedad, obsolescencia, factor de impacto, inmediatez, actualidad temática, aislamiento, autocitación, coeficiente general de citación, temática, descripción, paradigma adoptado, teoría desde la que se trabaja, diseño, riesgos muestrales y técnicas de análisis (Flores-Fernández y Aguilera-Eguía, 2020).

La base de datos multidisciplinar más utilizada para la construcción de indicadores bibliométricos, en ciencias básicas y experimentales, es el Science Citation Index, perteneciente al Web of Science Group, Clarivate, y que incluye aproximadamente 5.300 revistas. Otras bases de importancia en las distintas áreas del saber, son: MEDLINE (Index Medicus), PASCAL (Bibliographie Internacional), BIOSIS (Biological Abstracts), Chemical Abstracts, CAB Internacional, COMPENDEX (Engineering Index), INSPEC (Physics Abstracts) y MathSci (Mathematical Science). Dadas las limitaciones que el uso de las bases internacionales presentan para Latinoamérica, particularmente en cuanto a visibilidad, idioma y contextualización, se ha desarrollado una serie de bases de datos regionales para recabar información sobre sus publicaciones científicas y tecnológicas, entre las que se encuentran: LILACS (Literatura de América Latina y el Caribe en Ciencias de la Salud), CLASE (catálogos en línea de la Dirección General de Bibliotecas de la Universidad Nacional Autónoma de México), AGRIS (Organización de Alimentos y Agricultura de la Organización de las Naciones Unidas), LATINDEX (Sistema Regional de Información en Línea para Revistas Científicas de Iberoamérica), SciELO (Scientific Electronic Library Online), Redalyc (Red de Revistas Científicas de Acceso Abierto), REDIB (Red Iberoamericana de Innovación y Conocimiento Científico) y LatinREV (Red Latinoamericana de Revistas en Ciencias Sociales). En el caso venezolano, con el pasar del tiempo se han creado y desaparecido sistemas de 
evaluación de revistas e investigadores, como: publicaciones científicas y tecnológicas acreditadas por el Fondo Nacional de Ciencia, Tecnología e Innovación (FONACIT), Revistas Venezolanas de Ciencia y Tecnología (REVENCYT), Programa de Promoción del Investigador (PPI) y Programa de Estímulo a la Innovación e Investigación (PEII).

Entre las limitaciones relacionadas con la aplicación de indicadores globales a Latinoamérica, se encuentran por ejemplo, las estrategias adoptadas por Estados Unidos de América, Inglaterra, Canadá y otros países desarrollados, donde se ha convertido al proceso de publicación científica en una industria que garantiza el dominio de indicadores como visibilidad, citación e influencia intelectual (Romero-Torres et al., 2013), soportada además en las políticas académicas del campo laboral en universidades y centros de investigación. Para reducir esta brecha, se hace necesaria la adopción de indicadores no convencionales adaptados a las realidades científicas, tecnológicas y socio-económicas de nuestros países, como: ponderación de la visibilidad, creación de cuartiles para nuevos indicadores, combinación de métodos cuantitativos y cualitativos (Gregorio-Chaviano, 2018), aplicación al entorno regional o local, especificidades por áreas de conocimiento, entre otros, garantizando de esta manera un equilibrio en las evaluaciones y el reconocimiento de calidad para nuestras instituciones, revistas e investigadores.

\section{Dr. Julio César Marín L.}

Editor ejecutivo

Revista Técnica de la Facultad de Ingeniería - LUZ

\section{Referencias bibliográficas}

De Filippo, D., Fernández, M. T. (2003). Bibliometría: importancia de los indicadores bibliométricos. En: El estado de la ciencia, principales indicadores de ciencia y tecnología iberoamericanos/interamericanos. Ed. Albornoz, M. Buenos Aires: Red de Indicadores de Ciencia y Tecnología Iberoamericana e Interamericana (RICYT), 69-76.

Flores-Fernández, C., Aguilera-Eguía, R. (2020). Indicadores bibliométricos y su importancia en la investigación clínica. ¿Por qué conocerlos? Revista de la Sociedad Española del Dolor, 26(5), 315-316.

Gregorio-Chaviano, O. (2018). Evaluación y clasificación de revistas científicas: reflexiones en torno a retos y perspectivas para Latinoamérica Revista Lasallista de Investigación, 15(1), 166-179.

Peralta, M., Frías, M., Chaviano, O. (2015). Criterios, clasificaciones y tendencias de los indicadores bibliométricos en la evaluación de la ciencia. Revista Cubana de Información en Ciencias de la Salud, 26(3), 290-309.

Romero-Torres, M., Acosta-Moreno, L. A., Tejada-Gómez, M. A. (2013). Ranking de revistas científicas en Latinoamérica mediante el índice $h$ : estudio de caso Colombia. Revista Española de Documentación Científica, 36(1), e003. 